Théologiques

Théologiques

\title{
La référence à la Tradition dans la production d'une « théologie morale » : illustration d'un cas
}

\section{André Guindon}

Volume 1, numéro 2, octobre 1993

Les textes fondateurs. Entre autorité et liberté

URI : https://id.erudit.org/iderudit/602391ar

DOI : https://doi.org/10.7202/602391ar

Aller au sommaire du numéro

Éditeur(s)

Faculté de théologie de l'Université de Montréal

ISSN

1188-7109 (imprimé)

1492-1413 (numérique)

Découvrir la revue

Citer cet article

Guindon, A. (1993). La référence à la Tradition dans la production d'une " théologie morale " : illustration d'un cas. Théologiques, 1(2), 63-78.

https://doi.org/10.7202/602391ar
Résumé de l'article

La production d'un discours éthique dans un domaine aussi inexploré que la nudité donne l'occasion d'examiner à neuf la référence à la Tradition dans une telle entreprise. Une approche " éthique » des textes fondateurs n'y a produit que du matériau apte à construire une histoire des moeurs de dénudation. Une approche " théologique », en accueillant l'expérience de Dieu chez les témoins chrétiens, identifie l'autorité propre à la Tradition dans le discours éthique. Révélation des « moeurs divines », la Tradition guide le discernement des pratiques morales de dénudation (ou de tout autre agir moral) qui sont de nature à initier chrétiennes et chrétiens à l'Ineffable Mystère. 


\section{La référence à la Tradition dans la production d'une "théologie morale": illustration d'un cas.}

André GUINDON

Faculté de Théologie Université Saint-Paul

\section{RÉSUMÉ}

La production d'un discours éthique dans un domaine aussi inexploré que la nudité donne l'occasion d'examiner à neuf la référence à la Tradition dans une telle entreprise. Une approche "éthique» des textes fondateurs n'y a produit que du matériau apte à construire une histoire des moeurs de dénudation. Une approche "théologique", en accueillant l'expérience de Dieu chez les témoins chrétiens, identifie l'autorité propre à la Tradition dans le discours éthique. Révélation des "moeurs divines", la Tradition guide le discernement des pratiques morales de dénudation lou de tout autre agir moral) qui sont de nature à initier chrétiennes et chrétiens à l'Ineffable Mystère.

\section{Introduction}

Posons d'entrée de jeu que la "théologie morale" se distingue du "discours éthique" par sa référence obligée à la Tradition. Je soutiendrai que celle-ci fonde très exactement une "théologie" de l'agir humain, non une éthique révélée. Je construirai ma démonstration à partir d'un cas en éthique sectorielle. Celui-ci offrira aussi l'occasion 
d'illustrer en quel sens serait acceptable l'expression "éthique chrétienne".

\section{Construire une éthique du dénuder 1}

En 1991, j'entrepris l'exploration du domaine du vêtir et du dénuder ${ }^{2}$. Ces pratiques relèvent de la "culture matérielle", un domaine pour lequel les sciences humaines se sont récemment passionnées. En ces gestuelles quotidiennes serait encodé tout le Zeitgeist d'une époque. L'éthique, au contraire, a déserté ce champ depuis les ravages qu'y semèrent les moralistes qui militèrent, au début du siècle, dans les "croisades pour la modestie". Malgré leur horreur de la chair, ces victoriens eurent le mérite de soupçonner que la présence incorporée de soi-même à autrui représente une gestuelle de tous les instants et constitue la toile de fond de tous nos échanges. Réussirions-nous à déchiffrer le lexique et la syntaxe du vêtir et du dénuder que nous mettrions à jour un niveau primaire d'expression de soi qui affecte radicalement tous les autres. Ceci est particulièrement le cas de la gestuelle de dénudation qui met à l'épreuve l'ingénuité du corps. Pour le propos de cet article, nous nous confinerons à celle-ci.

L'absence d'un débat sur la moralité du dénuder nous dispose à considérer sans parti pris de vieilles questions méthodologiques que soulève la mise en oeuvre du discours éthique. Ici, tout était à inventer. Il fallait définir à neuf l'objet disciplinaire (de quelle nudité construire l'éthique?), en saisir le potentiel humanisant (en quoi l'humanité s'enrichit-elle par les gestuelles de dénudation?) et définir les conditions morales, personnelles et sociales, qui favorisent son actualisation du potentiel humain.

Selon l'acceptation la plus recevable du concept de "loi naturelle", la tradition catholique postule que l'expérience morale est un

\footnotetext{
${ }^{1}$ La langue française n'offre pas de mot pour signifier l'agir qui consiste tout à la fois à «se mettre nu» et à «se conduire nu». Je prête donc à «dénuder» un sens élargi pour inclure l'agir en état de nudité. Par contre, «nudité», qui signifie l'état d'une personne nue, a acquis un sens actif: «le fait de vivre nu». J'utilise donc les deux mots indifféremment, avec une préférence pour «dénuder» qui évoque davantage l'«agir". objet propre du discours éthique.

2 Je suis redevable aux assistants de recherche Christine Jamieson et Kevin Murphy pour leur précieuse collaboration. Celle-ci a été rendu possible grâce à la subvention 410-91-0071 du Conseil de recherches en sciences humaines du Canada.
} 
dénominateur assez commun du genre humain pour que ses évidences de base jouissent d'une certaine universalité. Fort de cette conviction, je me suis immergé dans des études anthropologiques, historiques, littéraires et psychosociales des gestuelles de dénudation à travers le temps et l'espace afin de saisir la façon dont ce phénomène a été interculturellement vécu, compris, jugé. Contrairement à ce qu'on aurait pu prédire à partir du "sens commun" propre à notre culture, les définitions du signifiant de la nudité foisonnent ${ }^{3}$. Innombrables sont les façons d'“être nu». Construction culturelle, la nudité des uns est souvent imperceptible aux autres. Elle se définira ici par l'exhibition des organes génitaux, là par celle des pieds ou des lèvres ou des mains ou du nombril ou des cuisses ou même du seul gland du pénis ou des seules lèvres intérieures de la vulve. Une parure symbolique - une ficelle autour de la taille - pourra démarquer la femme vêtue de la femme nue. L'objet du discours éthique sur le dénuder se définira donc moins par le signifiant que par le signifié qui introduit destinateurs et destinataires à l'univers du sens.

Si, dans des mythes d'origine, nudité et vêtement pouvaient, à l'instar du cru et du cuit, représenter un système d'opposition entre ce qui précède l'état de culture et ce qui en procède ${ }^{4}$, cette logique n'est plus opératoire telle quelle dans les sociétés historiques. Chimérique que ce retour à la "nature", au régime du cru, des dénuders d'êtres enculturés. Aucune société connue - exception faite de tribus dégénérescentes 5 - ne vit la nudité comme fait brut, insignifiant, inconscient. Aussi les signifiants du dénuder ne renvoient-ils pas, comme on le fait valoir dans la littérature anti-nudiste, à un retour à la nature ou à l'animalité. Ils renvoient à des signifiés culturels remarquablement homogènes d'une société à l'autre. On pourrait ergoter

3 Les apologètes occidentaux du nudisme n'ont généralement pas perçu combien occidentale est leur définition de la nudité à partir du dévoilement des organes génitaux. Voir, par exemple, Marc-Alain DESCAMPS, Le nu et le vêtement. Paris, Éditions Universitaires, 1972, 23-28; Vivre nu: psychosociologie du naturisme. Paris, Éditions Trismégiste, 1987, 17-18; Paul ABLEMAN, Beyond Nakedness. Los Angeles, Elysium Growth Press, 1985, 7.

4 Claude LEVI-STRAUSS, L'homme nu. Paris, Plon, 1971, notamment: 44, 304-308, $323,347-348,351,454$, a confirmé cette congruence entre cru /nu et cuit/ vêtu dans l'anthropologie structurale des peuplades indiennes des Amériques.

5 Ibid: 30. Comme Claude LEVI-STRAUSS l'expliquait dans Le cru et le cuit. Paris, Plon, 1964, 152, il s'agit alors d'une gestuelle déshumanisée plutôt que non-encorehumanisée, comme la putréfaction marque, dans les mythes tupi-guarani, la transformation «sauvage» du cru. 
sur leur nombre, leurs nuances et l'agencement le plus apte à dégager leur signification. $\dot{A}$ toutes fins utiles, je les ai liés en faisceaux autour de trois concepts binaires: bien-être/mal-aise, simplicité/duplicitéhumiliation, union /désunion-abus.

Ayant constaté que, indépendemment des sagesses et des croyances qui les animent, les peuples ont reconnu dans les gestes de dénudation, l'une ou l'autre version de ces signifiés "enculturants" / «déculturants", il restait à préciser les conditions morales qui favoriseraient l'éclosion des premiers et l'évitement des seconds. Au plan personnel, intégration, authenticité et bienveillance. Au plan social, la responsabilité et ses multiples ramifications. Cette tâche complétée, j'estimai mon travail éthique terminé.

\section{Contestation "chrétienne» du discours éthique}

Les données qui avaient contribué à définir l'objet, à en faire sens et à en établir les conditions d'accomplissement moral provenaient aussi de cultures influencées par le christianisme. Je n'y ai cependant rien décelé que je n'avais repéré ailleurs. Que des signifiants originaux de nudité et les énoncés prescriptifs qui s'y rapportent aient pu caractériser des sociétés d'inspiration chrétienne n'infirme pas cette assertion. D'une part, ces codes ont varié considérablement, jusqu'à se contredire, d'une société chrétienne à l'autre et d'une époque à la suivante. D'autre part, on ne parvient jamais à établir quelle considération spécifiquement chrétienne motiverait telle ou telle directive morale concernant la gestuelle de nudité. Lorsqu'elles sont exposées, les motivations rejoignent celles qu'on retrouve en dehors du christianisme.

Avec la naissance du naturisme dans l'Allemagne du XIXe siècle, cependant, et sa diffusion en Europe et aux États-Unis, la littérature chrétienne adoptera un ton polémique. Racisme et nudophobie y firent leur apparition ${ }^{6}$. Si la majorité des théologiens moralistes argumentaient à partir de considérations éthiques, leur opposition revêtait souvent un caractère doctrinal. Le nudisme, selon eux, serait

6 Voir, par ex., Edgar HOCEDEZ, «Pour la modestic chrétienne», Nouvelle Revue Théologique 52 (1925) 401; Edgar JANSSENS, Pudeur et nudisme. Liège, La Pensée Catholique, 1931, 5; Ludwig RULAND, Pastoral Medicine. St. Louis, B. Herder. 1936, 258. 
une erreur 7 , un culte irréconciliable avec les idéaux chrétiens 8 et catholiques 9 . On ne s'embarrassait guère d'expliquer comment la foi chrétienne était compromise dans des "colonies nudistes" caractérisées, à l'époque, par des moeurs quasi-puritaines 10 . À une exception près: Erik Peterson, patrologue de bon renom, qui, en 1934, condamna le nudisme au nom d'une métaphysique théologique ${ }^{11}$. Selon lui, la gestuelle du vêtir et du dénuder toucherait aux vérités les plus centrales de la foi. "ll est significatif - c'est inclus, à vrai dire, dans la nature des choses - que tous les cercles qui font, à quelque degré, campagne en faveur du nu, le font avec des motifs idéologiques et religieux qui s'opposent consciemment à l'enseignement révélé de l'Église" 12. Cette déclaration percutante est suivie d'une étude du thème de la nudité et du vêtement chez les Pères de l'Église.

À la lumière de l'étude de Peterson ${ }^{13}$, on pourrait donc penser que si le travail éthique auquel je m'étais livré pouvait avoir quelque mérite dans le cadre d'une "éthique humaniste", chrétiennes et chrétiens ne sauraient être dupes. Ils savaient "quelque chose" à propos de la nudité et de sa moralité que personne d'autres ne soupçonnent. Aussi fallait-il

7 Johannes STEIZENBERGER, Précis de morale chrélienne. Toumai, Desclée, 1960, 259; Fritz TILLMANN, The Master Calls: A Handbook of Christian Living. Baltimore, Helicon Press, 1960, 202-203.

8 Bernard HÄRING, The Law of Christ: Moral Theology for Priests and Laity. Cork, The Mercier Press, 1961-1967, vol. III: 232.

9 E. HOCEDEZ, «Pour la modestic...», 402; Edwin F. HEALY, Moral Guidance: A Textbook in Principles of Conduct for Colleges and Universities. Chicago, Loyola University Press, 1943, 203; Henry DAVIES, Moral and Pastoral Theology: A Summary. London, Sheed and Ward, 1952, 76.

10 William E. HARTMAN, Marilyn FITHIAN ct Donald JOHNSON, Nudist Society: The Controversial Study of the Clothes-Free Naturist Movement in America. éd. rev., Los Angeles, Elysium Growth Press, 1991, 59-70.

11 Erik PETERSON, «Theologie des Kleides», Benediktinisches Monatschrift 16 (1934) 347-356. Y. M.-J. Congar en fit une traduction, «Pour une théologie du vêtement», La Vie Spirituelle: Supplément 46 (1936) 168-179. Elle fut encore publicee en 1943 à Lyon, Éditions de l'A beille, coll. La Clarté-Dieu, VIII, 23 p. Les renvois sont à la publication de 1943.

12 E. PETERSON, Pour une théologie..., 5-6.

13 Sebastian P. BROCK, "Clothing Metaphors as a Means of Theological Expression in Syriac Tradition", dans Typus, Symbol, Allegorie bei den östlichen Vätern und ihren Parallelen im Mittelalter. (SCHMID, M. et M. F. GEYER, éds), Regensburg, F. Pustet, 1982, 11-40, ne fait que fournir des textes de la tradition syrienne sans remettre en cause l'interprétation de Peterson . 
examiner la contestation "chrétienne" du discours éthique telle qu'elle se présente dans l'article de Peterson ${ }^{14}$.

\section{L'“argument d'autorité» de Peterson}

Les textes patristiques étudiés portent sur deux lieux connexes dans l'“exégèse spirituelle» des Pères: la nudité mythique d'Adam et Eve et celle des néophytes au baptême. Lorsqu'ils évoquent la condition originelle des protoparents, argumente Peterson, les Pères affirment que leurs corps étaient "vêtus de la gloire de Dieu". Aussi ne voyaientils pas leur nudité. Le péché ayant ruiné cette tenue paradisiaque, le statut métaphysique du corps humain en fut modifié. En les chassant de l'Éden, Yahwé Dieu fit pour eux des tuniques de peau dont il les revêtit ( $G n$ 3, 21). Indices de honte, ces tuniques exprimaient toutefois leur quête de l'habit resplendissant des origines. La quête aboutit au baptême chrétien lorsque les néophytes reçoivent le "vêtement blanc".

Sans ignorer la signification symbolique de ce vêtement de gloire perdu et retrouvé, Peterson en fait néanmoins une application littérale. Autant son insistance sur la honte des corps en l'absence du vêtement reçu au baptême que sa déclaration initiale concernant le nudisme fondé nécessairement ("jedoch im Sachverhalt als solchen gegeben") sur une idéologie hérétique écartent une interprétation purement métaphorique. C'est bien parce qu'ils prétendent que, même dévêtus de leurs habits matériels, ils se tiennent encore en présence de Dieu, que les nudistes et leurs défenseurs doivent être confondus. On ne leur connaît pas d'autre hérésie que cette gestuelle de dénudation communale.

L'étude attentive du dossier suggère qu'il y a glissement de la métaphore à la réalité et déformation des vues exprimées par les Pères. D'abord, ceux-ci n'entretiennent aucune ambiguïté concernant le statut du "vêtement de gloire". Métaphorique, cette expression ne dénote pas la réalité telle qu'elle existe physiquement. C'est, au contraire, la nudité des êtres paradisiaques qui fonctionne comme "vêtement de gloire". Dans leur souci d'humanisation, les Latins insistent sur l'honnêteté de leurs esprits qui ne voyaient rien d'obscène. Épris de divinisation, les Grecs invoquent le regard bienveillant de Dieu qui crée la confiance

14 Une étude détaillée de ce dossier paraîtra dans Laval philosophique et théologique. Je n'en reprendrai ici que les conclusions. Notons que l'article de Peterson est encore cité régulièrement comme l'autorité en la matière dans les travaux qui abordent le thème de la nudité et du vêtement dans la Tradition chrétienne. 
nécessaire pour une gestuelle de nudité conviviale. Tels des enfants, Adam et Eve jouaient sans inhibition devant Dieu, leur Père aimant 15.

Des tuniques matérielles dans les récits d'origine, les Pères n'ont guère retenu que la tunique de peaux fabriquée par Dieu. Dans l'interprétation orthodoxe 16 , elle devient costume de honte, occultant le corps pécheur et mortel. Plusieurs Pères voient donc dans le vêtir une oeuvre de duplicité, de mensonge, de tromperie et de luxure. Aussi, d'anciens rituels baptismaux prévoyaient-ils qu'avant de se présenter au baptême, le néophyte - comme d'anciens rituels orientaux l'exigeront du candidat à la profession monastique 17 - se dépouillait de cette ancienne tunique de peaux et la piétinait en signe de renonciation au "vieil homme" chassé du Paradis 18. Du vêtement matériel, les Pères prêchent une morale de péché, non de grâce.

Finalement, l'insistance de Peterson sur le fameux "vêtement blanc" déplace l'accent des rites baptismaux anciens. Loin d'appartenir aux rites essentiels, celui-ci est lié à la participation à l'Eucharistie. Pour les rites essentiels, l'immersion et la réception du Saint-Esprit, les initiés des deux sexes - et même le prêtre qui préside, selon $L a$ Tradition Apostolique 19 - descendent nus dans la piscine. "Ô merveille!" s'exclame Cyrille de Jérusalem, "vous étiez nus sous les yeux de tous et vous n'en aviez point de honte». Pourquoi cette candeur? "C'est qu'en vérité vous offriez l'image de notre premier père Adam qui, au paradis terrestre, était nu et sans honte" 20. "Se dépouiller du vieil homme" (Col 3,10-11), c'est abandonner la corruption dont les habits mondains sont un symbole. "Se revêtir du

15 Robert MURRAY, Symbols of Church and Kingdom: A Study in Early Syriac Tradition. Londres, Cambridge University Press, 1975, 304-306, a montré que ce thème de l'enfance d'Adam et Eve est fréquent chez les Pères prénicéens et chez les Syriens.

16 Sur cette question, voir Pier Franco BEATRICE «Le tuniche di pelle - Antiche letture di Gen 3, 21», dans La tradizione dell'enkratia. (BIIANCHI, U., éd.), Rome, Edizioni dell'Ateneo, 1985, 433-484.

17 Pierre RAFFIN, Les rituels orientaux de la profession monastique. (Spiritualité orientale, 4), Bégrolles, Abbaye de Bellefontaine, 1968, 37 et 158-159.

18 Jonathan Z. SMITH, «Garments of Shame», IIistory of Religions 5 (1966) 224 233.

19 Apostoliké Paradosis: The Treatise of the Apostolic Tradition of S. Hippolytus of Rome, 215 et 11. (DIX, G. , éd), $2^{\mathrm{e}}$ éd., Londres, SPCK, 1968, 33 et 35.

20 CYRILLE DE JÉRUSALEM, Catéchèses mystagogiques, 2, 2, dans L'initiation chrétienne. (HAMMAN, A., ćd.), Paris, B. Grasset, 1963, 41. 
Christ" (Ga 3,27-28), c'est porter son "costume d'Adam", sa glorieuse nudité telle que créée par Dieu et restaurée par le Christ. Si la métaphore du vêtement symbolise théologiquement la relation de grâce qui unit les protoparents, puis les baptisés au Seigneur, les réalités humaines, qu'évoquent en même temps les Pères, fonctionnent à l'inverse: la nudité corporelle irradie à la fois l'innocence humaine et la bienveillance divine alors que le vêtement matériel est entreprise d'iniquité.

\section{Résultats d'une approche éthique des Textes}

À peine soixante ans passés, donc, un spécialiste des Pères de l'Église scrutait les Textes pour appuyer de leur autorité, au delà "de pures raisons de moralité" 21 , une polémique anti-nudiste "chrétienne". À une époque où l'exhibition de bras nus était considérée comme une abomination, son enquête excluait a priori certains éléments de réponse. Cercle herméneutique dont résultaient la glorification du vêtir et la réprobation du dénuder.

L'atténuation du tabou a permis de détecter dans les Textes des Pères des dénuders acceptables, voire hautement idéalisés. L'attitude morale de ceux-ci envers la nudité réelle n'est pas pour autant obvie. Ils vivaient dans une société où le costume féminin et masculin exposait davantage le corps nu que le système vestimentaire d'aujourd'hui en Occident. La vie quotidienne comportait par ailleurs un ensemble imposant de "nudités coutumières" dont l'Europe occidentale n'a vu le déclin qu'au XVIle siècle. On se dénudait en commun pour se baigner, se coucher, se soulager, accomplir certains travaux. La conversatio civilis mettait en contact avec la nudité statutaire des enfants, des esclaves, des prisonniers. Au $\|{ }^{e}$ siècle, Cyprien ne s'objectait qu'au dénuder des vierges chrétiennes qui, dans les bains publics mixtes de Carthage, risquaient de perdre leur dignité corporelle (honor corporis) 22 . Au Ve siècle, lors du "premier synode de saint Patrick", les évêques d'Irlande stipulaient que

tout clerc, du portier au prêtre, qui se montre sans tunique [longue] et qui ne couvre pas la honte et la nudité de son corps,

21 E. PETERSON, Pour une théologie..., 5.

22 De Habitu Virginum, 19. (BOUTET, J. ćd.), Paris, Descléc, 1926, 50-51. 
dont les cheveux ne sont pas taillés selon la coutume romaine, et dont la femme circule la tête dévoilée, doit, ainsi que sa femme, être méprisé par les fidèles et renvoyé de l'Église 23.

Nudité mal séante que le péripatéticien Théophraste d'Érèse, habitué pourtant à la gymnité grecque, reprochait au rustre qui, "[e]n s'asseyant, [...] retrousse son manteau au-dessus du genou, sans souci de laisser apercevoir sa nudité" 24 .

Non seulement l'univers sensible dans lequel ces Textes ont été écrits nous est-il inaccessible, mais le climat moral dans lequel ils ont été rédigés est difficile à reconstituer. Ainsi, I'histoire de l'éthique sexuelle prétendument chrétienne a été pensée à partir de la notion romantique d'un monde pré-chrétien imaginé comme l'Éden du nonrefoulé. Les communautés chrétiennes auraient élaboré une éthique de renonciation sexuelle en réaction à la débauche qui aurait prévalu dans les classes cultivées de l'Empire. La recherche historique contemporaine a discrédité cette vue 25 . Caractérisées par des moeurs sexuelles particulières liées à leur attente du retour imminent du Christ, ces communautés durent au contraire prouver qu'elles respectaient les normes sexuelles romaines et qu'elles aussi pratiquaient la pietas civique 26 . Problématique éthique totalement étrangère à la nôtre.

La connaissance par documents n'atteignant jamais qu'un aspect, parfois secondaire, du réel, nous ignorons aussi la représentativité des vues qui y sont exprimées en regard des moeurs communément pratiquées et approuvées dans les communautés chrétiennes du passé. Ainsi, les Textes patristiques sont didactiques et livrent les vues d'auteurs mâles issus d'une classe privilégiée et exerçant des fonctions d'autorité. Que représentent leurs directives sur tel ou tel geste de dénudation? Soucis politiques ou moraux? Réflexes patriarcaux ou éthiques? Règles de civilité patricienne ou de rationalité morale?

23 Synodus I Sancti Patricii, 6, L. BIELER, éd., The Irish Penitentials. (Scriptores Latini Hiberniae, 5), Dublin. Dublin Institute of Advanced Studies, 1963, 54-55.

24 Caractères 4, 7, (NAVARRE, Octave, ćd.), $2^{\mathrm{e}}$ éd. rev. et cor., Paris, Les Belles Lettres, 1952, 47.

25 Peter BROWN, The Body and Society: Men, Women and Sexual Renunciation in Early Christianity. New York, Columbia University Press, 1988, 21-22.

26 John Kevin COYLE, «Empire and Eschaton. The Early Church and the Question of Domestic Relationships», Église et Théologie 12 (1980) 35-94. 
Expérience ancienne de la nudité et contexte moral incommensurables avec les nôtres, compréhensions culturellement conditionnées, celles des auteurs comme celles des interprètes contemporains, autant de considérations qui mettent en cause l'autorité des Textes fondateurs dans la production d'un discours éthique contemporain sur le dénuder. Les approches éthiques des Sources offrent-elles autre chose que des études historiques de l'éthique? Au fur et à mesure que la recherche se poursuit, nous amassons de nouveaux renseignements sur la variété extraordinaire des moeurs de nudité qui sont apparues dans le christianisme et des jugements à leur propos. Basé sur les données que j'ai déjà colligées, je soupçonne que ces coutumes et leur appréciation ne divergeront pas de façon significative, pour autant que l'éthique est concernée, de celles qu'influencent d'autres religions ou sagesses.

\section{Une approche théologique des Témoins}

Pourtant, il existe dans les Églises chrétiennes une pratique ininterrompue des Sources dans le but d'éclairer les attitudes et les agirs moraux des croyants. Qu'espère-t-on y trouver exactement? Confirmations et exhortations divinement inspirées de ce que les moralistes philosophes ou de tradition religieuse non-chrétienne savent ou sont en mesure de savoir ${ }^{27}$ ? Pourquoi ce recours à la foi dans un domaine qui, comme la cosmologie ou la politique, est accessible au discernement humain? Dieu qui "a créé l'homme au commencement" ne l'a-t-il pas “laissé à son propre conseil?» (Si 15,14) 28 .

S'il y a une réponse satisfaisante à ces questions, elle doit se trouver dans une approche de la Tradition qui cherche à y entendre les Témoins de la foi en Dieu (He 11). Les préoccupations que ces Témoins peuvent manifester pour la régulation morale des pratiques nues n'ont guère d'intérêt pour elles-mêmes. Elles n'en acquièrent que dans la mesure où elles portent un témoignage sur la sorte de Dieu que des conduites spécifiques de nudité sont susceptibles de révéler aux croyants. Proprement théologique, cette approche nourrit la foi qui, par la médiation de Témoins $(P h 4,9 ; R m 10,17)$, découvre progressivement "ce que l'oeil n'a pas vu, ce que l'oreille n'a pas entendu, et ce qui n'est pas monté au coeur de l'homme, tout ce que Dieu a préparé pour ceux qui

27 Les Jésuites allemands Karl Rahner, Josef Fuchs et Bruno Schüller semblent n'y avoir guère cherché autre chose. Voir, William C. SPOHN, What Are They Saying About Scripture and Ethics. New York, Paulist Press, 1984, 36-53.

28 Les traductions bibliques sont celles de la TOB. 
l'aiment" (1Co 2,9). Indispensable témoignage, pour qui s'initie tant bien que mal aux "moeurs divines", que celui de celles et ceux qui ont entendu, vu, contemplé, touché de leurs mains afin que nous soyons en communion avec le Père et avec son Fils Jésus Christ (1 Jn 1,1-3).

Lorsque ces Témoins, dont le regard est fixé sur les mirabilia Dei, s'amènent à commenter les moeurs de nudité, tout ce qui peut y avoir de propos moral dans ces gloses est à ranger parmi les souvenirs de l'album de famille. Elles reflètent la façon dont nos Ancêtres, dans les circonstances qui étaient leurs, ont tranché au meilleur de leur connaissance les dilemmes moraux qui se posaient à eux. Libre à nous de juger que les principes qu'ils estimaient pertinents ne s'appliquent plus dans les gestuelles de nudité qui sont nôtres. Rudolf Bultmann suggérait qu'écouter un Texte plutôt que ce Dieu qui sans cesse nous invite à partager sa vie peut mener à la désobéissance 29 .

\section{Comment l'éthique est-elle chrétienne?}

L'éthique peut-elle donc être chrétienne? L'expérience de la recherche sur le dénuder me porte à conclure négativement si l'on entend par là que sa spécificité chrétienne lui viendrait du fait que des valeurs, des principes, des règles ou des codes moraux seraient "révélés" au peuple chrétien à travers ses médiateurs: Jésus, éminemment, mais aussi Moïse et les Prophètes, les Apôtres et leurs Successeurs, les Mères et les Pères de l'Église. D'humaine qu'elle est, l'éthique se fait toutefois chrétienne, suggèrerais-je, lorsque celles et ceux qui exercent le discernement moral et cherchent à le mettre en pratique sortent graduellement de l'idolâtrie et croissent dans l'Ineffable Mystère de Dieu tel qu'il est révélé par Jésus et les autres grands Témoins de la foi. Examinons ce rapport entre pratique d'humanité et pratique de divinité.

Donnée, la foi croît à même l'expérience humaine. En connaissance (credere Deo), d'abord, puisque celle-ci constitue l'ultime référent indispensable à tout discours humainement intelligible. La qualité de

29 Rudolf Karl BULTMANN, Jésus: mythologie el démythologisation. Paris, Seuil, 1968, 80-90, 93-94. Sandra M. SCHNEIDERS, «Living Word or Dead(ly) Letter. The Encounter between the New Testament and Contemporary Experience», The Catholic Theological Society of America Proceedings 47 (1992) 59, estime même que certains textes scripturaires sont devenus «irrémédiablement immoraux en regard de certaines des questions les plus importantes de notre temps». 
notre propre expérience morale nous prédispose à mieux saisir dans la foi tout ce que les Témoins rapportent sur ces "réalités que l'on ne voit pas" (He 11,1). Avant de connaître Dieu face à face, bien sûr, "nous voyons dans un miroir et de façon confuse" (1 Co 13,12). Simple miroir que ces réalités soumises à notre expérience humaine. Ne point les regarder, pourtant, nous condamnerait à ne rien voir du tout. "Lieu du savoir éthique", affirme René Simon ${ }^{30}$, la conduite humaine l'est aussi d'un certain savoir théologique. Les personnes qui se réfugient dans «la piété" pour s'évader de leur responsabilité humaine se condamnent, paradoxalement, à adorer une idole.

L'expérience humaine sert encore à la foi pour ébaucher son élan vers Dieu et pour raffermir graduellement son lien de connaissance confiante avec Lui (credere in Deum). Cette affirmation prend pour acquis que la réalité de ce monde n'existe pas dans un no man's land en dehors de Jésus-Christ révélant le Père dans l'Esprit. Aussi la participation à la réalité divine est-elle inséparable de la participation à la réalité du monde créé 31 . Les Sources, justement, révèlent ce "milieu divin" dans lequel s'inscrit notre existence morale. Si l'éthique ne descend pas du ciel, comme l'admettent de plus en plus les théologiens moralistes de la Tradition protestante eux-mêmes ${ }^{32}$, je suggère qu'elle $y$ monte ${ }^{33}$. En somme, l'éthique est "chrétienne" en ce qu'elle offre un substrat humain à la croissance dans le Mystère de la vie divine. L'agir humain devient, par grâce, agir chrétien ouvert sur un avenir absolu en Dieu. Il se convertit en "pratique divine", participation initiatique à la vie propre de Dieu. Pierre Arribard écrit dans la même veine que la Bible cindique des chemins de conversion du moral au théologal et ouvre

30 René SIMON, Fonder la morale. Paris, Seuil, 1974, 200.

31 Dietrich BONHOEFFER, Éthique. Genève, Labor et Fides, 1965, 56-61, notamment: 59 .

32 Voir, par ex., James M. GUSTAFSON, Theology and Christian Ethics. Philadelphia, Pilgrim Press, 1974, 129-130; Dietrich BONHOEFFER, «Questions fondamentales d'une éthique chrétienne», dans Textes choisis. (GRUNOW, R., éd.), Paris/Genève, Centurion/Labor et Fides, 1970, 55.

33 Jean-Marc CHARRON, De Narcisse à Jésus: La quête de l'identité chez François d'Assise. Montréal/Paris, Paulines/Cerf, 1992, 60, cite un texte de La légende des trois compagnons selon lequel «ses vertus naturelles [il s'agit de François] furent le premier échelon d'où il s'éleva à un [certain] point de grâce...». 
à la réalisation d'un parcours théologique dans le domaine de l'agir humain. 34 .

Reste à illustrer cette position pour qu'on ne nous accuse pas de verbiage. Retournons au cas d'espèce: de quelle(s) façon(s) une pratique morale de nudité serait-elle apte à initier les personnes chrétiennes qui s'y adonnent à l'Ineffable Mystère? En d'autres mots, comment les personnes qui deviennent plus humaines en pratiquant des formes de dénuder qui les aident à mieux intégrer spiritualité et corporalité, à s'exprimer plus véridiquement aux autres et à mieux partager leur intimité avec eux..., comment ces personnes seraient-elles en mesure de croître dans leur foi?

Vécu comme bien-être sous la régulation de normes morales propres à intégrer les valeurs de l'esprit à l'existence incorporée, le dénuder sera pédagogique du Mystère confessé dans le premier article du Symbole de la foi qui proclame la divinité créatrice "du ciel et de la terre". Les croyants savent conceptuellement qu'il n'y a pas de place dans le Credo pour un démiurge malveillant, architecte de réalités physiques mauvaises. Leurs comportements face à la nudité seront pourtant plus instructifs à ce propos que la récitation de formules dogmatiquement correctes. Pensons à l'exibition de la nudité de Jésus par les artistes de la Renaissance occidentale. Leo Steinberg a démontré que cette expérience positive de la nudité constitue un énoncé de foi convainquant - et une catéchèse iconographique - sur l'ahumanation" du Christ et, par conséquent, sur la bonté intégrale de la corporalité humaine 35 . Bien ambigu, au contraire, cet énoncé dogmatiquement correct récité en 1925 par le moraliste jésuite Edgar Hocedez: “le catholicisme n'est pas l'ennemi du corps", alors que tout ce qui précède polémique contre les nudistes qui tentent de "réhabiliter la chair" 36 . Expression spontanée, de la part de Hocedez, d'une expérience humaine de honte envers une chair si irrémédiablement souillée que sa vue éveillerait les passions les plus abjectes. Dans ces conditions, comment le rapport créatural pourrait-il correspondre effectivement à celui qu'énonce la formule orthodoxe?

34 Pierre ARRIBARD, «La référence à l'Écriture en vue de l'agir des croyants: le point de vue d'un moraliste», dans Écriture et pratique chrétienne. (Lectio divina, 96), Paris, Cerf, 1978,31 .

35 Léo STEINBERG, La sexualité du Christ dans l'art de la Renaissance et son refoulement moderne. Paris, Gallimard, 1978, $265 \mathrm{p}$.

36 E. HOCEDEZ, «Pour la modestie...», 405 et 411. 
Reconnue dans sa bonté et dans sa beauté, portée avec dignité et respect, vécue en harmonie avec la nature, la nudité humaine est susceptible, au contraire, d'initier les personnes qui ont la foi à l'Ineffable Mystère d'une divinité dont les opérations n'ont aucune affinité avec le mal.

Vécue comme simplicité sous la régulation de normes morales propres à favoriser le dire authentique de soi, la gestuelle de dénudation est apte à initier chrétiennes et chrétiens à un autre aspect de l'Ineffable Mystère. Avec Maurice Zundel nous la qualifierons de "divine Pauvreté" 37. Pauvreté des divines Personnes qui ne s'approprient la vie divine qu'en la rapportant tout entière aux Autres. Pauvreté de Personnes qui, en leur Trinité, ne "possèdent" rien puisque chacune est éternellement reçue des Autres. Brisure décisive que ce Dieu "pauvre" avec un Dieu de pouvoir et de domination, d'opacité et de déception, une idole construite à même nos insécurités et nos insincérités.

Zundel attribue à François d'Assise l'intuition bouleversante de la divine Pauvreté. Si François choisit pour épouse Dame Pauvreté, c'est qu'elle symbolise et favorise le dépouillement du coeur. Ascèse, certes, mais surtout mystique de la transparence, d'un moi réceptif à la communication de l'altérité. Ouvrir en soi l'espace qui permet à autrui de le combler de sa présence. Cette intuition fondamentale, le Poverello l'aurait dramatisée à Assise en réinventant la vénérable gestuelle de dénudation (de renonciation au moi "mondain") monastique. On sait que des Pères et aussi des Mères (par exemple, la très célèbre Marie l'Égyptienne ${ }^{38}$ ) du désert ont suivi à la lettre l'aphorisme de Jérôme: Nudus nudum Christum sequi 39.

37 Maurice ZUNDEL, L'Évangile intérieur. $2^{\mathfrak{c}}$ éd., Saint-Maurice/Paris, Oeuvres St. Augustin/Desclée de Brouwer, 1939, 51-59; La pierre vivante. Paris, Les Éditions Ouvrières, 1954, 61-79; Retraite aux Franciscaines de Lons-le-Saulnier à Ghazir (Liban), texte polycopić, 1959, 82-94; Morale et mystique. Bruges, Desclée de Brouwer, 1962, 43-77; Quel homme et quel Dieu? Retraite au Vatican. Paris, Fayard. $1976,130-142$.

38 Jacques LACARRIÈRE, Les hommes ivres de Dieu. (Signes des temps, XII), Paris, B. Arthaud, 1961, 193-197; Margaret Ruth MILES, Carnal Knowing: Female Nakedness and Religious Meaning in the Christian West. Boston, Beacon Press, 1989, 63-66.

39 Voir le dossier compilé par Matthäus BERNARDS, «Nudus nudum Christum sequi», Wissenschaft und Weisheit 14 (1951)148-151. 
Nudités coutumières non-compliquées, non-affectées, portées avec l'ingénuité des gestes quotidiens, nudités ménagées dans des existences délibérément soucieuses de présence simple, véridique, dépouillée des feintes conventionnelles, nudités de transparence mystique, autant de lieux d'une gestuelle initiatique à I'Ineffable Mystère de la divine Pauvreté.

Vécue comme intimité partagée, sous la régulation de normes propres à assurer la bienveillance des intentions et des conduites, la gestuelle de dénudation se prête à initier à l'Ineffable Mystère de l'Amour libérateur. Qui ne connaît l'admirable "Dieu est amour" de la première épître de Jean $(4,8$ et 16)? Qui ne sait qu'il suffit donc, pour résumer tout ce qu'ont pu prescrire lois et prophètes, d'aimer Dieu et le prochain comme soi-même (Mt 22,34-40;MC 12,28-34;LC 10,25-28)? Pourtant Jonathan Swift constatait que nous, chrétiens, "avons juste assez de religion pour nous haïr, mais pas assez pour nous aimer les uns les autres" 40 . Une religion qui relie effectivement à une divinité semeuse de zizanie, d'hostilité et de peur ${ }^{41}$. Pourquoi pas de celle qui attache au Dieu Amour de l'énoncé de foi? Pas plus que la récitation des énoncés corrects sur Dieu Créateur ou sur Dieu Trinité, celle sur Dieu Amour ne suffit à le rendre présent amoureusement dans la vie des personnes baptisées. Seule la pratique dans la foi du partage amoureux authentique permet d'accueillir en nos vies le Dieu dont l'amour libère au lieu d'asservir $(1 \mathrm{Jn} 3,18 ; 4,8.20$ et 21). L'amour humain est signe de croissance dans la communion intime avec Dieu et celle-ci rejaillit en retour sur la qualité divine de celui-là.

Nudités privées qui, dans l'intimité amoureuse, symbolisent la totalité du don, nudités communautaires qui, dans des contextes favorisant la confiance - une "fiance du coeur" en l'à-venir de nos relations humaines - disent la solidarité de personnes dépouillées des oripeaux de leurs avoirs et de leurs compétences, nudités d'une convivialité entre des êtres humains qui ne sont que ce qu'ils sont, nudités offertes à voir dans une altérité si fragile et pourtant si réelle. Autant de gestuelles susceptibles d'initier à la communion avec un Dieu Amour radicalement différent des idoles qui asservissent despotiquement à leurs ridicules décrets les créatures qui les adorent.

40 Jonathan SWIFT, «Pensées sur divers sujets», dans Oeuvres. (La Pléiade), Paris, Gallimard, 1988, 569.

41 Qu'on pense au dossier accablant monté par Jean DELUMEAU, Le péché et la peur: La culpabilisation en Occident (xviie-xviiie siècles). Paris, Fayard, 1984, 741 p. 
Faut-il ajouter que le dénuder, en sa matérialité, ne crée aucune valeur morale? Faut-il rappeller, avec les sagesses les plus diverses, que par vice individuel ou collectif, la nudité peut servir des projets déshumanisants et, par là même, est susceptible d'initier ses adeptes à des cultes religieux idolâtriques? II suffisait ici de suggérer de quelles façons possibles une expression nue circonspecte et honnête de soi à soimême et à autrui peut contribuer, chez celles et ceux qui ont la foi, à une expérience plus authentique de ce Dieu que manifestent les Témoins de la Tradition.

\section{Conclusion}

Le cas de la gestuelle de nudité, de par sa singularité même et par l'absence de controverses éthiques actuelles à son sujet, se prête bien à illustrer en quel sens fonctionne la référence à la Tradition dans la production d'une "théologie morale". La référence à des "directives morales" dans les Textes de la Tradition en vue de régler nos agirs m'apparaît invalide. Elle ne dégage pas - comme nous l'avons vérifié dans le cas du dénuder - de discernements moraux qui diffèrent en quoi que ce soit de ceux que tiennent les autres sagesses humaines et religieuses de l'humanité. Les études qui se réclament d'une telle référence pour juger de nos conduites - comme celle de Peterson contre la pratique nudiste de son temps - illustrent la complexité des problèmes autant exégétiques qu'herméneutiques auxquels elles sont confrontées. Toujours à reprendre, elles ne nous éclairent que sur l'histoire de l'éthique chrétienne.

Par contre, la référence à la Tradition est constitutive de la "théologie morale" lorsqu'elle porte sur le Dieu de Jésus-Christ proclamé par les Témoins et dont la foi des chrétiennes et des chrétiens cherche à pénétrer l"Ineffable Mystère. Dans sa fonction "morale", la théologie s'efforce de discerner pour le temps présent les attitudes et les pratiques morales qui sont les plus aptes à initier les êtres humains que nous sommes aux "moeurs divines". 